\title{
Chloride administration in the intensive care unit, an independent predictor of mortality
}

\author{
S Smolders ${ }^{1 *}$, RJ Bosman², H Endeman ${ }^{2}$ \\ From ESICM LIVES 2015 \\ Berlin, Germany. 3-7 October 2015
}

\section{Introduction}

Chloride is the body's most important extracellular anion, important in many physiologic processes including acid base balance and osmotic pressure [1]. Hyperchloremic metabolic acidosis is a common finding in critically ill patients and may be associated with renal failure and even increased mortality [2].

\section{Objectives}

The objective of our study was to determine if chloride administration in a critically ill population is associated with hospital mortality.

\section{Methods}

We performed a retrospective observational study in a 24 bed tertiary mixed medical surgical ICU in a teaching hospital in the Netherlands. Patients admitted to the ICU in the period between January 1th 2008 and November 1 th 2014 were screened for eligibility. Inclusion criteria were: 1 . $\geq 18$ years. 2 . Length of stay (LOS) of $\geq 72$ hours. Readmissions to the ICU were excluded. The primary end points for our analysis was hospital mortality. Univariate analysis was performed with Wilcoxon rank sum test for nonparametric data. Multivariate analysis was performed with predictors of ICU and hospital mortality (age, Acute Physiology and Chronic Health Evaluation IV predicted mortality) as well as factors associated with (hyper)chloremia, metabolic acidosis and fluid resuscitation. Chloride administration was defined as the total amount of chloride in $\mathrm{mmol} / \mathrm{l}$ administered to a patient in a given time period.

\section{Results}

We studied 1471 patients. Mean age at admission was 67 years, APACHE II score 24, the average stay 10,5 days (table 1, baseline characteristics). In univariate analysis chloride levels at 72 hours were predictors of hospital mortality as were $\mathrm{pH}$, chloride administration and total fluid administration (table 2). A multivariate analysis was performed to determine whether chloride, chloride administration, fluid administration and $\mathrm{pH}$ are independent predictors of mortality. This models shows that chloride levels are not an independent predictor of hospital

Table 1. Baseline characteristics.

\begin{tabular}{llll}
\hline & Whole population Mean & Hospital survivors Mean & Non survivors Mean \\
\hline Age (years) & $67 \pm 12$ & $67 \pm 13$ & $69 \pm 12$ \\
\hline Sex (male) & $64,9 \%$ & $65,7 \%$ & $62,6 \%$ \\
\hline APACHE II Score & $24 \pm 7$ & $22 \pm 7$ & $28 \pm 7$ \\
\hline Admission type medical & $59 \%$ & $53,1 \%$ & $74,3 \%$ \\
\hline Admission type surgical & $41 \%$ & $46,9 \%$ & $25.7 \%$ \\
\hline Length of stay at ICU (hours) & 254 & 224 & 329 \\
\hline Acute renal failure & $22.9 \%$ & $20,4 \%$ & $29,3 \%$ \\
\hline CPR before admission & $12.3 \%$ & $9,9 \%$ & $18,6 \%$ \\
\hline Renal replacement therapy during ICU stay & $30.6 \%$ & $25,9 \%$ & $42,8 \%$ \\
\hline
\end{tabular}

'Onze Lieve Vrouwe Gasthuis, Pediatric Intensive Care Unit, Amsterdam, 
Table 2. Univariate analysis

\begin{tabular}{llll}
\hline & Hospital survivors Mean & Non-survivors Mean & P \\
\hline Chloride at admission (mmol/l) & $106,6 \pm 6,3$ & $106,8 \pm 6,5$ & 0,986 \\
\hline Chloride at 72 hours (mmol/l) & $106,3 \pm 5,1$ & $107,6 \pm 5,1$ & 0,000 \\
\hline pH at admission & $7,33 \pm 0,09$ & $7,30 \pm 0,10$ & 0.000 \\
\hline pH at 72 hours & $7,44 \pm 0,06$ & $7,41 \pm 0,07$ & 0.000 \\
\hline Total fluid administered at 24 hours (liters) & $4,04 \pm 2,6$ & $4,41 \pm 2,8$ & 0.027 \\
\hline Total fluid administered at 72 hours (liters) & $6,27 \pm 3,5$ & $7,58 \pm 4,1$ & 0.000 \\
\hline Chloride administered at 24 hours (mmol) & $507 \pm 306$ & $566 \pm 341$ & 0.006 \\
\hline Chloride administered at 72 hours (mmol) & $809 \pm 425$ & $809 \pm 425$ & 0.000 \\
\hline
\end{tabular}

Table 3. Multivariate analysis

\begin{tabular}{|c|c|c|c|c|c|c|c|c|c|}
\hline & \multicolumn{9}{|c|}{ Hospital mortality } \\
\hline & \multicolumn{3}{|c|}{24 Hour } & \multicolumn{3}{|c|}{48 Hour } & \multicolumn{3}{|c|}{72 Hour } \\
\hline & $\begin{array}{l}\text { Odd } \\
\text { ratio }\end{array}$ & $\begin{array}{l}\text { Confidence } \\
\text { interval }\end{array}$ & $\begin{array}{l}P \\
\text { value }\end{array}$ & $\begin{array}{l}\text { Odd } \\
\text { ratio }\end{array}$ & $\begin{array}{l}\text { Confidence } \\
\text { interval }\end{array}$ & $\begin{array}{l}P \\
\text { value }\end{array}$ & $\begin{array}{l}\text { Odd } \\
\text { ratio }\end{array}$ & $\begin{array}{l}\text { Confidence } \\
\text { interval }\end{array}$ & $\begin{array}{l}P \\
\text { value }\end{array}$ \\
\hline \multicolumn{10}{|l|}{ Variable } \\
\hline $\begin{array}{l}\text { APACHE } 4 \text { predicted } \\
\text { mortality }\end{array}$ & 14.352 & $8.84-23.31$ & 0.000 & 14.6 & $8.88-24.31$ & 0.000 & 11.74 & $7.51-18.35$ & 0.000 \\
\hline Age & 1.023 & $1.010-1.035$ & 0.000 & 1.022 & $1.009-1.035$ & 0.001 & 1.026 & $1.014-0.782$ & 0.000 \\
\hline Total fluid administration & 0.635 & $0.443-0.908$ & 0.013 & 0.654 & $0.486-0.881$ & 0.005 & 0.613 & $0.480-0.782$ & 0.000 \\
\hline Chloride loading & 1.004 & $1.001-1.007$ & 0.013 & 1.004 & $1.001-1.006$ & 0.003 & 1.005 & $1.003-1.007$ & 0.000 \\
\hline$\overline{\mathrm{pH}}$ & 0.072 & $0.009-0.579$ & 0.013 & 0.018 & $0.002-0.167$ & 0.000 & 0.006 & $0.001-0.047$ & 0.000 \\
\hline Chloride & 1.004 & $0.977-1.032$ & 0.763 & 1.020 & $0.990-1.051$ & 0.188 & 1.003 & $0.998-1.052$ & 0.071 \\
\hline
\end{tabular}

mortality, chloride administration however is an independent predictor (OR 1.005; 95\% CI 1.003-1.007 at 72 hours) as is $\mathrm{pH}$, total fluid administration and age (table 3 ).

\section{Conclusions}

In conclusion, chloride administration in a critically ill population is an independent risk factor for mortality, even when corrected for total fluid administration, $\mathrm{pH}$ and chloride levels. Further studies the optimal resuscitation fluid with regard to chloride levels are needed.

\section{Authors' details}

'Onze Lieve Vrouwe Gasthuis, Pediatric Intensive Care Unit, Amsterdam, Netherlands. ${ }^{2}$ Onze Lieve Vrouwe Gasthuis, Intensive Care Unit, Amsterdam, Netherlands.

Published: 1 October 2015

\section{References}

1. Powers F: The role of chloride in acid-base balance. J Intraven Nurs 1999 22:286-91

2. Berend $\mathrm{K}$, et al: Chloride: The queen of electrolytes? European Journal of Internal Medicine 2012, 23:203-211.

\section{doi:10.1186/2197-425X-3-S1-A975}

Cite this article as: Smolders et al.: Chloride administration in the

intensive care unit, an independent predictor of mortality. Intensive Care Medicine Experimental 2015 3(Suppl 1):A975.

\section{Submit your manuscript to a SpringerOpen ${ }^{\circ}$ journal and benefit from:}

- Convenient online submission

- Rigorous peer review

- Immediate publication on acceptance

- Open access: articles freely available online

- High visibility within the field

- Retaining the copyright to your article

Submit your next manuscript at $>$ springeropen.com 